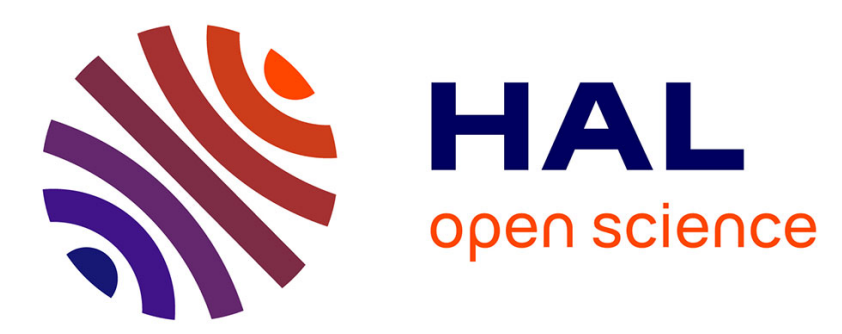

\title{
The stability of the fractional Volterra integro-differential equation by means of $\Psi$-Hilfer operator revisited
}

Dumitru Baleanu, Reza Saadati, José Vanterler

\section{To cite this version:}

Dumitru Baleanu, Reza Saadati, José Vanterler. The stability of the fractional Volterra integrodifferential equation by means of $\Psi$-Hilfer operator revisited. 2020. hal-03012059

\section{HAL Id: hal-03012059 \\ https://hal.science/hal-03012059}

Preprint submitted on 18 Nov 2020

HAL is a multi-disciplinary open access archive for the deposit and dissemination of scientific research documents, whether they are published or not. The documents may come from teaching and research institutions in France or abroad, or from public or private research centers.
L'archive ouverte pluridisciplinaire HAL, est destinée au dépôt et à la diffusion de documents scientifiques de niveau recherche, publiés ou non, émanant des établissements d'enseignement et de recherche français ou étrangers, des laboratoires publics ou privés. 


\title{
The stability of the fractional Volterra integro-differential equation by means of $\Psi$-Hilfer operator revisited
}

\author{
Dumitru Baleanu ${ }^{1}$, Reza Saadati ${ }^{2, *}$ José Vanterler da C. Sousa ${ }^{3}$
${ }^{1}$ Department of Mathematics, Cankaya University, 06530 Balgat, Ankara, Turkey and Institute of Space Sciences, P.O. Box, MG 23, R 76900, Magurele-Bucharest, Romania
${ }^{2}$ Department of Mathematics, Iran University of Science and Technology, Narmak, Tehran, Iran
${ }^{3}$ Department of Applied Mathematics, Institute of Mathematics, Statistics and Scientific \\ Computing - Imecc, São Paulo, Brazil
}

\begin{abstract}
In this note, we prove the Ulam-Hyers stability of a fractional Volterra integral equation. Our results modify the Theorem 4 of [9] and present a corrected proof with a modified approximation.
\end{abstract}

Keywords: Fixed point theorem, fractional Volterra integral equation, UlamHyers stability, $\Psi$-Hilfer fractional derivative.

\section{Introduction and Preliminaries}

Consider continuous map $\omega:[0, P] \rightarrow \Omega$ which $\Omega$ is a Banach space. In this paper, we apply a fixed point theorem (FPT) to study Ulam-Hyers stability (UH-stability) of the solution of the fractional Volterra integral equation

$$
\omega(\tau)=\zeta(\tau, \omega(\tau))+\mathcal{I}_{0+}^{\varrho} ; \mathcal{K}(\tau, \sigma, \omega(\sigma)) d \sigma,
$$

where $\mathcal{I}_{0^{+}}^{\varrho ;}$ is the $\Psi$-Riemann-Liouville fractional integral defined by

$$
\mathcal{I}_{0^{+}}^{\varrho ; \Psi} \omega(\tau)=\frac{1}{\Gamma(\varrho)} \int_{0}^{\tau} \mathcal{Q}_{\Psi}^{\varrho}(\tau, \sigma) \omega(\sigma) d \sigma
$$

where $\mathcal{Q}_{\Psi}^{\varrho}(\tau, \sigma):=\Psi^{\prime}(\sigma)(\Psi(\tau)-\Psi(\sigma))^{\varrho-1},[1]-[7]$, with $0<\varrho<1$, and $\zeta$ : $[0, P] \times \Omega \rightarrow \Omega, \mathcal{K}:[0, P]^{2} \times \Omega \rightarrow \Omega$.

We work on the weighted space $C_{1-\xi ; \Psi}[0, P]$ of continuous mappings $\omega$ which defined by $[7,9]$ and with norm $\|\cdot\|_{C_{1-\xi ; \Psi}[0, P]}$.

${ }^{*}$ Corresponding author. E-mail: rsaadati@eml.cc 
For a continuous map $\omega(\tau)$, satisfying

$$
\left\|\zeta(\tau, \omega(\tau))+\mathcal{I}_{0^{+}}^{\varrho ; \Psi} \mathcal{K}(\tau, \sigma, \omega(\sigma)) d \sigma-\omega(\tau)\right\|_{C_{1-\xi ; \Psi}} \leq \psi(\tau)
$$

with $\psi(\tau)>0$ and $0 \leq \tau \leq P$, let we can find a solution $\nu(\tau)$ of (1.1) such that

$$
\|\omega(\tau)-\nu(\tau)\|_{C_{1-\xi ; \Psi}[0, P]} \leq K \psi(\tau),
$$

for some $K>0$, so (1.1) has the UH-stability. Note that (1.1) can be considered as integral equation

$$
\omega(\tau)=\zeta(\tau, \omega(\tau))+\frac{1}{\Gamma(\varrho)} \int_{0}^{t} \mathcal{Q}_{\Psi}^{\varrho}(\tau, \sigma) \mathcal{K}(\tau, \sigma, \omega(\sigma)) d \sigma
$$

In Proposition 1 of [9] the authors proved that if $\left(\Omega,\|\cdot\|_{C_{1-\xi ; \Psi}}\right)$ is a normed space, then the function $\delta: \Omega \times \Omega \rightarrow \mathbb{R}, \delta(\omega, \nu)=\|\omega-\nu\|_{C_{1-\xi ; \Psi}}$ is a metric on $\Omega$. By [10] every normed space induce a metric by equality $\delta(\omega, \nu)=\|\omega-\nu\|$, then Proposition 1 of [9] is obvious and no need any proof.

The next theorem introduced by Diaz and Margolis [11] and used by Cădariu and Radu for studying the concept of stability of functional equations [12, 13, 14].

Theorem 1.1 (Alternative FPT). Let $(\Gamma, \delta)$ be a complete $[0, \infty]$-valued metric space. Assume that $\Lambda: \Gamma \times \Gamma$ is a strictly contractive operator with the Lipschitz constant $\mathcal{L}<1$. If there exists a nonnegative integer $k$ such that $\delta\left(\Lambda^{k+1} \omega, \Lambda^{k} \omega\right)<1$, for some $\omega \in \Gamma$. Thus

1. $\left\{\Lambda^{n} \omega\right\}$ converges to a FP $\omega^{*}$ of $\Lambda$.

2. $\Lambda \omega^{*}=\omega^{*}$, also $\omega^{*}$ is the unique in

$$
\Gamma^{*}=\left\{\nu \in \Gamma ; \delta\left(\Lambda^{k} \omega, \nu\right)<\infty\right\} .
$$

3. If $\nu \in \Gamma^{*}, \delta\left(\nu, \omega^{*}\right) \leq \frac{1}{1-\mathcal{L}} d(\Lambda \nu, \nu)$.

\section{Ulam-Hyers stability}

In the proof of Theorem 4 of [9] the authors defined the mapping, $\Lambda: \mathcal{M} \rightarrow \mathcal{M}$, by

$$
\begin{aligned}
& \Lambda(\omega(\tau)) \\
& =\mathcal{I}_{0^{+}}^{\varrho ;}\left[\zeta(\tau, \omega(\tau))+\frac{1}{\Gamma(\varrho)} \int_{0}^{t} \mathcal{Q}_{\Psi}^{\varrho}(\tau, \sigma) \mathcal{K}(\varsigma, \sigma, \omega(\sigma)) d \sigma\right] \\
& =\mathcal{I}_{0^{+}}^{\varrho ; \Psi}[\zeta(\tau, \omega(\tau))]+\mathcal{I}_{0^{+}}^{\varrho ; \Psi}\left[\frac{1}{\Gamma(\varrho)} \int_{0}^{t} \mathcal{Q}_{\Psi}^{\varrho}(\tau, \sigma) \mathcal{K}(\varsigma, \sigma, \omega(\sigma)) d \sigma\right]
\end{aligned}
$$


which is not suitable for fractional integral equations. More exactly, since by Theorem 1.1 the fixed point of $\Lambda$ is the unique solution of (1.1) and the operator $\Lambda$ helps to find a solution for integral equation (1.1), so could not be an integral operator as above, then the authors of [9] could not find an accurate approximation. In this section we present a correct proof by defining a suitable function $\Lambda: \mathcal{M} \rightarrow \mathcal{M}$.

Theorem 2.1. Assume that $\Omega$ is a Banach space and $\mathcal{L}, \mathcal{L}_{1}, \mathcal{L}_{2}$, and $P$ are positive constants for which $0<\mathcal{L}_{1}+\mathcal{L}_{2} \mathcal{L}<1$. Let $\zeta:[0, P] \times \Omega \rightarrow \Omega$, $\mathcal{K}:[0, P]^{2} \times \Omega \rightarrow \Omega$ and continuous map $\psi:[0, P] \rightarrow(0, \infty)$ satisfying

$$
\begin{gathered}
\|\zeta(\tau, \omega)-\zeta(\tau, \nu)\|_{C_{1-\xi ; \Psi}} \leq \mathcal{L}_{1}\|\omega-\nu\|_{C_{1-\xi ; \Psi}[0, P]} \\
\|\mathcal{K}(\tau, \sigma, \omega(\sigma))-\mathcal{K}(\tau, \sigma, \nu(\sigma))\|_{C_{1-\xi ; \Psi}} \leq \mathcal{L}_{2}\|\omega-\nu\|_{C_{1-\xi ; \Psi}[0, P]}
\end{gathered}
$$

and

$$
\frac{1}{\Gamma(\varrho)} \int_{0}^{\tau} \mathcal{Q}_{\Psi}^{\varrho}(\tau, \sigma) \psi(\sigma) d \sigma \leq \mathcal{L} \psi(\tau)
$$

for all $\tau, \sigma \in[0, P]$, and $\omega, \nu \in \Omega$. If the continuous map $\eta:[0, P] \rightarrow \Omega$ satisfying

$$
\left\|\eta(\tau)-\zeta(\tau, \eta(\tau))-\frac{1}{\Gamma(\varrho)} \int_{0}^{\tau} \mathcal{Q}_{\Psi}^{\varrho}(\tau, \sigma) \mathcal{K}(\tau, \sigma, \eta(\sigma)) d \sigma\right\|_{C_{1-\xi ; \Psi}[0, P]} \leq \psi(\tau),
$$

$0 \leq \tau \leq P$, thus we can find a unique continuous map $\eta_{0}:[0, P] \rightarrow \Omega$ such that for every $\tau \in[0, P]$,

$$
\eta_{0}(\tau)=\zeta\left(\tau, \eta_{0}(\tau)\right)+\frac{1}{\Gamma(\varrho)} \int_{0}^{\tau} \mathcal{Q}_{\Psi}^{\varrho}(\tau, \sigma) \mathcal{K}\left(\tau, \sigma, \eta_{0}(\sigma)\right) d \sigma
$$

and

$$
\left\|\eta(\tau)-\eta_{0}(\tau)\right\|_{C_{1-\xi ; \Psi}} \leq \frac{1}{1-\left[\mathcal{L}_{1}+\mathcal{L}_{2} \mathcal{L}\right]} \psi(\tau) .
$$

Proof. Set $\mathcal{M}=\{\omega:[0, P] \rightarrow \Omega, \omega$ is continuous $\}$ and define a mapping $\delta$ : $\mathcal{M}^{2} \rightarrow[0, \infty]$ by

$$
\delta(\omega, \nu)=\bigwedge\left\{\mathcal{C} \geq 0:\|\omega(\sigma)-\nu(\sigma)\|_{C_{1-\xi ; \Psi}} \leq \mathcal{C} \psi(\sigma), 0 \leq \sigma \leq P\right\} .
$$

In $[9]$ and $[12,13,14]$ the authors proved that $(\mathcal{M}, \delta)$ is a complete $[0, \infty]$-valued metric space. Consider $\Lambda: \mathcal{M} \rightarrow \mathcal{M}$, such that

$$
\Lambda(\omega(\tau))=\zeta(\tau, \omega(\tau))+\frac{1}{\Gamma(\varrho)} \int_{0}^{\tau} \mathcal{Q}_{\Psi}^{\varrho}(\tau, \sigma) \mathcal{K}(\tau, \sigma, \omega(\sigma)) d \sigma .
$$


Suppose $\omega, \nu \in \Omega, \mathcal{C}_{\omega \nu} \in[0, \infty]$, and $\delta(\omega, \nu) \leq \mathcal{C}_{\omega \nu}$. So, for every $\tau \in[0, P]$,

$$
\|\omega(\tau)-\nu(\tau)\|_{C_{1-\xi ; \Psi}}<\mathcal{C}_{\omega \nu} \phi(t) .
$$

Using (2.1) to (2.3), we get

$$
\begin{aligned}
& \|\Lambda \omega(\tau)-\Lambda \nu(\tau)\|_{C_{1-\xi ; \Psi}} \\
& \leq\|\zeta(\tau, \omega)-\zeta(\tau, \nu)\|_{C_{1-\xi ; \Psi}}+\| \mathcal{I}_{0^{+}}^{\varrho ; \Psi}\left(\mathcal{K}(\varsigma, \sigma, \omega(\sigma))-\mathcal{K}(\varsigma, \sigma, \nu(\sigma)) \|_{C_{1-\xi ; \Psi}}\right. \\
& \leq \mathcal{L}_{1}\|\omega(\tau)-\nu(\tau)\|_{C_{1-\xi ; \Psi}}+\mathcal{I}_{0^{+}}^{\varrho ; \Psi} \mathcal{L}_{2}\|\omega(\tau)-\nu(\tau)\|_{C_{1-\xi ; \Psi}} \\
& \leq \mathcal{L}_{1} \mathcal{C}_{\omega \nu} \psi(\tau)+\mathcal{L}_{2} \mathcal{I}_{0^{+}}^{\rho ; \Psi}\left[\mathcal{C}_{\omega \nu} \psi(\sigma)\right] \\
& \leq\left[\mathcal{L}_{1}+\mathcal{L}_{2} \mathcal{L}\right] \mathcal{C}_{\omega \nu} \psi(\tau) .
\end{aligned}
$$

Then we have

$$
\delta(\Lambda \omega, \Lambda \nu) \leq\left[\mathcal{L}_{1}+\mathcal{L}_{2} \mathcal{L}\right] \delta(\omega, \nu),
$$

which implies that the contractively property of $\Lambda$, because $0<\mathcal{L}_{1}+\mathcal{L}_{2} \mathcal{L}<1$. Since $\eta \in \mathcal{M}$ and using (2.4), we get

$$
\begin{aligned}
& \|\Lambda \eta(\tau)-\eta(\tau)\|_{C_{1-\xi ; \Psi}} \\
& =\left\|\zeta(\tau, \omega(\tau))+\frac{1}{\Gamma(\varrho)} \int_{0}^{\tau} \mathcal{Q}_{\Psi}^{\varrho}(\tau, \sigma) \mathcal{K}(\tau, \sigma, \omega(\sigma)) d \sigma-\eta(\tau)\right\|_{C_{1-\xi ; \Psi}} \\
& \leq \psi(\tau),
\end{aligned}
$$

and so,

$$
\delta(\Lambda \eta, \eta) \leq 1<\infty .
$$

Now, using Theorem 1.1 let us, to find a unique map $\eta_{0} \in \mathcal{M}^{*}=\{\nu \in \mathcal{M}$ : $\delta(\Lambda \eta, \nu)<\infty\}$ such that $\Lambda \eta_{0}=\eta_{0}$ and so

$$
\eta_{0}(\tau)=\zeta\left(\tau, \eta_{0}(\tau)\right)+\mathcal{I}_{0^{+}}^{\varrho ; \Psi}\left[\mathcal{K}\left(\varsigma, \sigma, \eta_{0}(\sigma)\right)\right] .
$$

Using Theorem 1.1 and (2.8), we have

$$
\delta\left(\eta, \eta_{0}\right) \leq \frac{1}{1-\left[\mathcal{L}_{1}+\mathcal{L}_{2} \mathcal{L}\right]} d(\Lambda f, f) \leq \frac{1}{1-\left[\mathcal{L}_{1}+\mathcal{L}_{2} \mathcal{L}\right]},
$$

which implies that (2.6). Set

$$
\beta=\frac{1}{1-\left[\mathcal{L}_{1}+\mathcal{L}_{2} \mathcal{L}\right]} .
$$

Consider another continuous map $\theta$ satisfying (2.5) and (2.6). Thus, $\eta \in \mathcal{M}$, $\delta(\eta, \theta)<\beta$, and

$$
\theta(\tau)=\zeta(\tau, \theta(\tau))+\frac{1}{\Gamma(\varrho)} \int_{0}^{\tau} \mathcal{Q}_{\Psi}^{\varrho}(\tau, \sigma) \mathcal{K}(\tau, \sigma, \theta(\sigma)) d \sigma .
$$


We prove that, $\theta \in \mathcal{M}^{*}$ is a FP of $\Lambda$.

By (2.10), we have $\Lambda \theta=\theta$. Now, we are ready to prove that $\delta(\Lambda \eta, \theta)<\infty$. Using (2.10) and $\delta(\eta, \theta)<\beta$, imply that

$$
\begin{aligned}
& \|\Lambda \eta(\tau)-\theta(\tau)\|_{C_{1-\xi ; \Psi}} \\
= & \left\|\zeta(\tau, \eta(\tau))+\frac{1}{\Gamma(\varrho)} \int_{0}^{\tau} \mathcal{Q}_{\Psi}^{\varrho}(\tau, \sigma) \mathcal{K}(\tau, \sigma, \eta(\sigma)) d \sigma-\eta(\tau)+\eta(\tau)-\theta(\tau)\right\|_{C_{1-\xi ; \Psi}} \\
\leq & \left\|\zeta(\tau, \eta(\tau))+\frac{1}{\Gamma(\varrho)} \int_{0}^{\tau} \mathcal{Q}_{\Psi}^{\varrho}(\tau, \sigma) \mathcal{K}(\tau, \sigma, \eta(\sigma)) d \sigma-\eta(\tau)-\eta(\tau)\right\|_{C_{1-\xi ; \Psi}} \\
& +\|\eta(\tau)-\theta(\tau)\|_{C_{1-\xi ; \Psi}} \\
\leq & (1+\beta) \psi(\tau),
\end{aligned}
$$

which implies that $\delta(\Lambda \eta, \theta) \leq 1+\beta<\infty$.

\section{References}

[1] Kilbas AA, Srivastava HM, Trujillo JJ. Theory and Applications of Fractional Differential Equations. Amsterdam: Elsevier; 2006.

[2] Oldham K, Spanier J. The Fractional Calculus Theory and Applications of Differentiation and Integration to Arbitrary Order. New York-London: Elsevier; 1974.

[3] Podlubny I. Fractional Differential Equations, Mathematics in Science and Engineering. San Diego: Academic Press; 1999.

[4] Samko SG, Kilbas AA, Marichev OI. Fractional integrals and derivatives. Theory Appl, Gordon Breach, Yvrdon. 1993; 1993:44.

[5] Katugampola UN. A new approach to generalized fractional derivatives. Bull Math Anal Appl. 2014; 6(4): 1-15.

[6] Almeida R. A Caputo fractional derivative of a function with respect to another function. Commun Nonlinear Sci Numer Simul. 2017; 44: 460-481.

[7] C. Sousa J, Oliveira E. On the $\Psi$-Hilfer fractional derivative. Commun Nonlinear Sci Numer Simul. 2018; 60: 72-91.

[8] C. Sousa J, Oliveira E. On a $\Psi$-fractional integral and applications. Compt Appl Math. 2019; 38: 4.

[9] Sousa, J. V. da C.; Fabio, G. R. de Oliveira, E. C. Stability of the fractional Volterra integro-differential equation by means of $\Psi$-Hilfer operator. Math. Meth. Appl. Sci. 42 (2019), 3033-3043.

[10] Kreyszig, E. Introductory functional analysis with applications. Wiley Classics Library. John Wiley \& Sons, Inc., New York, 1989. 
[11] Diaz, J. B.; Margolis, Beatriz. A fixed point theorem of the alternative, for contractions on a generalized complete metric space. Bull. Amer. Math. Soc. 74 (1968), 305-309.

[12] Cădariu, Liviu; Radu, Viorel. Fixed points and the stability of Jensen's functional equation. JIPAM. J. Inequal. Pure Appl. Math. 4 (2003), no. 1, Article 4, 7 pp.

[13] L. Cădariu, V. Radu, On the stability of the Cauchy functional equation: a fixed point approach. Grazer Math. Ber. 346 (2004), 43-52.

[14] L. Cădariu, V. Radu, Fixed point methods for the generalized stability of functional equations in a single variable. Fixed Point Theory Appl. 2008, Art. ID 749392 (2008). 